\title{
LXXIX. Researches in undulatory theory of light, in continuation of former papers
}

\author{
John Tovey Esq.
}

To cite this article: John Tovey Esq. (1836) LXXIX. Researches in undulatory theory of light, in continuation of former papers, Philosophical Magazine Series 3, 9:56, 420-429, DOI: 10.1080/14786443608649035

To link to this article: http://dx.doi.org/10.1080/14786443608649035

册 Published online: 01 Jun 2009.

Submit your article to this journal $\lceil\pi$

Џll Article views: 2

Q View related articles 5 
internus), or run in grooves (as the long head of the biceps), or perforate other tendons (as the deep flexor of the fingers', or turn through fibrous pulleys (as the digastric, the extensor of the toes, \&c.). By comparing the effect of a known force acting on particular tendons, at first in their natural situations, and afterward detached and free, the influence of friction in each case would be readily determined. This source of error seems to have been very generally overlooked by writers on animal mechanics.

I conclude, for the present, with suggesting that to distinguish the pectoralis major into "portio elevans" or "attollens," and "portio deprimens," might serve to impress the rationale of its peculiar insertion and twofold action, upon the memory of the student.

LXXIX. Researches in the Undulatory Theory of Light, in continuation of former Papers. By JoH Tovey, Esq.

To the Editors of the Philosophical Magazine and Journal.

Gentlemen,

HAVING deduced, (p. 500 of your last volume,) by a new method, the laws of the propagation of plane and spherical waves in elastic media, I will now, with your permission, show how the formulæ may be extended to the most simple cases which are known to occur in the undulatory theory of light, of waves not spherical emanating from a center of agitation.

(1.) It will be remembered that in my paper at p. 270 of the last volume, the sums $\Sigma$ were considered as comprised in three classes, when it appeared that those of the first class, composed of odd products of the differences, vanish, in consequence of the first supposition there made respecting the arrangement of the molecules. The sums also of the second class, composed of even products involving odd powers of the differences, were neglected; because the terms of these sums must be about half of them positive and half negative, and consequently the sums themselves very small in comparison with those of the third class, which last, being composed of even powers of the differences, have their terms all positive.

(2.) If the radius of the sphere of influence be not very much greater than the intervals between the molecules, the sums may or may not be sensibly the same for different directions of the coordinates, according as the intervals are the same or different for the different directions. Suppose, for example, every eight adjacent molecules to be at the corners of a rectangular parallelopiped; suppose fig. 1 to be a section of the medium, the dots denoting the molecules in their 
places of equilibrium : and suppose the circle to be a section of the sphere of the influence of the molecule which occupies its centre; then, the intervals between the molecules being greater in the horizontal than in the vertical direction, it is manifest that the sums in general will vary according to the directions of the coordinates, and that, when the planes of the coordinates are parallel to the planes which form the paral-

Fig. 1.

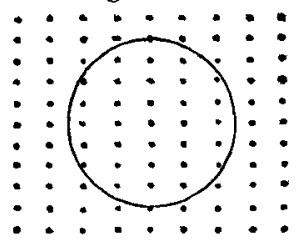
lelopipeds, the sums of the second class will have for every positive term an equal negative term, and consequently that these sums will vanish.

If the molecules of the æther and those of a transparent body form a compound vibrating medium, and if the observations just made be regarded as having reference only to the molecules of the body, the consequences will still be the same. (See paper at p. 270 of vol. viii.)

(3.) Since no light can be discovered to arise from the displacements $\xi^{*}$, we will neglect them, and then the equations (3.) of the paper at p. 7 of vol. viii. become

$$
\begin{aligned}
& \frac{d^{2} \eta}{d t^{8}}=m \Sigma\{\Phi(r) \Delta \eta+\psi(r)(\Delta y \Delta \eta+\Delta z \Delta \zeta) \Delta y\}, \\
& d^{z} \zeta=m \Sigma\{\phi(r) \Delta \zeta+\psi(r)(\Delta y \Delta \eta+\Delta z \Delta \zeta) \Delta z\} .
\end{aligned}
$$

Putting

$$
\begin{aligned}
& \Delta \eta=\frac{d \eta}{d x} \Delta x+\frac{d^{2} \eta}{d x^{2}} \cdot \frac{\Delta x^{2}}{2}+8 c . \\
& \Delta \zeta=\frac{d \zeta}{d x} \Delta x+\frac{d^{2} \zeta}{d x^{2}} \cdot \frac{\Delta x^{2}}{2}+8 c .
\end{aligned}
$$

and substituting these values in the previous equations, it is obvious that the principal sum of the second class (art. 1) is $\Sigma . \psi(r) \Delta x^{2} \Delta y \Delta z$. Now $y$ and $z$ may be taken in any directions which are perpendicular to $x$ and to one another; and if the directions be so chosen as to make this sum vanish, we may neglect the other sums of this class, and then the substitution will transform the equations into the second and third of the equations (2.) at p. 272 , and the velocities of the waves will be determined by the formulæ previously deduced in the paper at p. 5oo.

(4.) Suppose then $x_{1}, y_{1}, z_{i}$, to be rectangular coordinates, and the axis of $x_{1}$ to be fixed in the medium; suppose the plane of $x$ and $y$ to coincide with this axis; and suppose the molecules to be so arranged that the turning of the coordinates round it would not sensibly affect the values of the sums. In

* Airy's Math. Tracts, p. 340, art. $10 \mathrm{~J}$. 
this case the sum $\Sigma \cdot \psi(r) \Delta x^{2} \Delta y \Delta z$ (art. 3) will vanish : for let the plane of $x$ and $y$ pass through the molecule $n t$ and divide its sphere of influence into two hemispheres; then, since the arrangement of the molecules will, by the supposition, be sensibly the same in both, it follows that the terms of this sum will be half of them positive and half negative, and will destroy each ocher.

(5.) If we denote the length of the waves $\frac{2 \pi}{k}, \frac{2 \pi}{k_{l}}, \frac{2 \pi}{k_{l}}$, by $\lambda, \lambda_{l}, \lambda_{11}$; and their velocities $\frac{n}{k}, \frac{n_{l}}{k_{l l}}, \frac{n_{\| l}}{k_{l l}}$, by $v, v_{l}, v_{\| \prime}$; the equations (3.) of the paper at p. 500, give

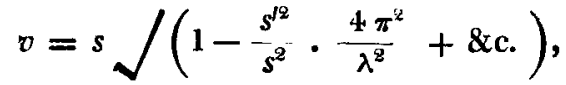

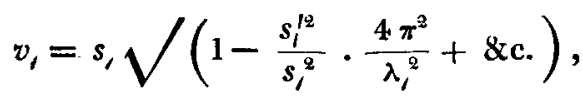

$$
\begin{aligned}
& v_{\|}=s_{\|} V\left(1-\frac{s_{\| \prime}^{\prime 2}}{s_{\| \prime}{ }^{2}} \cdot \frac{4 \pi^{2}}{\lambda_{\|}{ }^{2}}+8 \mathrm{c} .\right)
\end{aligned}
$$

(6.) Since our object is only to ascertain the forms of the wave-surfaces, we will, for the present, neglect the terms in these equations which depend upon the lengths of the waves, and suppose $v_{l}=s_{1}, v_{11}=s_{1 /}$; then, by the formulæ at p. 271, we have

$$
\begin{aligned}
& v_{1}^{2}=\frac{m}{2} \Sigma \cdot\left(\phi(r)+\psi(r) \Delta y^{2}\right) \Delta x^{2}, \\
& v_{l l}{ }^{2}=\frac{m}{2} \Sigma \cdot\left(\phi(r)+\psi(r) \Delta z^{2}\right) \Delta x^{2} .
\end{aligned}
$$

Now, let the axis of $z$ coincide with that of $z_{1}$, and let $\theta$ be the angle formed by the axes of $x$ and $x_{i}$; then, when $x, y, z$, and $x, y, z_{1}$, have the same origin, and are coordinates of the same molecule, we have, by the principles of analytical geometry,

and consequently,

$$
\begin{aligned}
& x=x_{1} \cos \theta-y_{1} \sin \theta, \\
& y=x_{1} \sin \theta+y_{l} \cos \theta, \\
& z=z_{j} ;
\end{aligned}
$$

$$
\begin{aligned}
& \Delta x=\Delta x_{1} \cos \theta-\Delta y_{1} \sin \theta, \\
& \Delta y=\Delta x_{1} \sin \theta+\Delta y_{1} \cos \theta, \\
& \Delta z=\Delta z_{1} .
\end{aligned}
$$

The last equations give

$$
\begin{aligned}
& \Delta x^{2}=\Delta x_{i}^{8} \cos ^{2} \theta+\Delta y_{l}^{2} \sin ^{2} \theta-2 \Delta x_{l} \Delta y_{l} \sin \theta \cos \theta, \\
& \Delta z^{2}=\Delta z_{l}^{2} ;
\end{aligned}
$$

and if we substitute these values in the second of the equations (3.), we have 


$$
\begin{gathered}
v_{u l}{ }^{5}=\frac{m}{2}\left\{\cos ^{2} \theta \Sigma \cdot\left(\phi(r)+\psi(r) \Delta z_{i}^{2}\right) \Delta x_{i}^{2}+\sin ^{2} \theta \Sigma\right. \\
\left.\cdot\left(\phi(r)+\psi(r) \Delta z_{i}^{2}\right) \Delta y_{i}^{2}\right\}
\end{gathered}
$$

$-m \sin \theta \cos \theta \Sigma .\left(\phi(r)+\psi(r) \Delta z_{l}^{2}\right) \Delta x_{1} \Delta y_{1}$.

The third sum in this equation must be zero in concequence of the supposed arrangement of the molecules round the axis of $x_{1}$, and therefore, if we denote by $c_{i}^{g}$ and $c_{11}^{y}$ the products of the first and second sums.multiplied by $\frac{m}{2}$, we have

$$
v_{\| 1}=\sqrt{ }\left(c_{1}^{2} \cos ^{2} \theta+c_{\| \prime}{ }^{2} \sin ^{2} \theta\right) \text {. }
$$

(7.) Let $\mathrm{C} \mathrm{D}, \mathrm{D} \mathrm{E}$, (fig. 2,) be elementary portions of a wave-surface diverging from the centre of agitation $O$; let $\mathrm{A} \mathrm{D}, \mathrm{B} \mathrm{E}$, be planes coincíding with $\mathrm{CD}, \mathrm{DE}$; and let $\mathrm{OA}, \mathrm{OB}$, be perpendiculars to these planes. Then the velocity with which the wave is, at $\mathrm{C} \mathrm{D}$, transmitted in the direction perpendicular to $\mathrm{CD}$, must be equal to the velocity of a plane wave moving in the direction of $\mathrm{OA}$; and the velocity with which the wave is, at $\mathrm{DE}$, transmitted in the direction perpendicular to $\mathrm{DE}$, must be the same as that of a plane wave moving in the direction of OB. Consequently, if we conceive an indefinite number of plane waves, which, at the commencenient of the time $t$, all pass through the centre of agitation $\mathrm{O}$, the wave surface will be that touched by all these plane waves at any instant.

(8.) Now let a number of planes like A D and BE, all perpendicular to the plane of the angle $x_{1}, \mathrm{O}_{1}$, be so drawn that their perpendicular distances from $O$, the origin of the coordinates, may be proportional to the values of $v_{\|}$given by the equation (4.); where $\theta$ is the angle which the perpendicular $x$ drawn from $O$ to any plane wave makes with the axis $\mathrm{O} x_{1}$. Then the curve in the plane of $x, \mathrm{O} y$, touched by all these planes will, by the property of the equation (4.), be an ellipse, the axes of which are proportional to $c$, and $c_{\mu}$.

(9.) The phænomena of chemistry show that molecular attractions and repulsions vary rapidly at particular distances of the molecules from each other. Suppose then the forces $m f(r)$, of the paper at p. 7 , to vary rapidly at particular values of $r$. The differential coefficients $\frac{d f(r)}{d r}$ may, in consequence, 
become, for these values, so large as to make the parts of the sums $\Sigma$ which contain them so much greater than the other parts, that the latter may be neglected. Accordingly we will assume this to be the case; and then the first of the equations (3.) becomes

$$
v^{2}=\frac{m}{2} \Sigma \cdot \psi(r) \Delta y^{2} \Delta x^{2} .
$$

This equation, being symmetrical with respect to $x$ and $y$, gives for $v$, the same value whether $x$ coincides with $\mathrm{O} x$, or with $\mathrm{O} y$, (fig. 2). We shall therefore assume that $v$, is sensibly the same for all values of $\theta$. And then if we put

we have

$$
\frac{m}{2} \Sigma \cdot\left(\phi(r)+\psi(r) \Delta y_{i}^{2}\right) \Delta x_{i}^{2}=c^{2}
$$

$$
v_{1}=c \text {. }
$$

(10.) Now conceive a number of plane waves, perpendicular to the plane of $x, \mathrm{O} y_{1}$, (fig. 2,) all of which, at the commencement of the time $t$, pass through the centre $\mathrm{O}$; and, since $v_{1}$ is the same for all values of $\theta$, conceive the velocities of these waves to be all equal; then their distances from the centre $\mathrm{O}$ will constantly be equal, and the curve, in the plane of $x, \mathrm{O}_{1}$, touched by all of them at any instant will be a circle.

(11.) If the system of coordinate planes be turned on the axis of $x$, the circle and ellipse (art. 10 and 8) will describe a sphere and spheroid. And since this turning of the coordinates will not, by the supposition (art. 4), sensibly affect the values of the sums, and consequently not alter those of $v$, and $y_{/}$, it follows that the agitation at the centre $O$ will in general produce two sets of waves; of which one set will be spheroidal, and the other spherical : the vibrations in the spheroidal waves being perpendicular to the axis of $x_{1}$, and the vibrations in the spherical waves perpendicular to those in the spheroidal.

(12.) From the supposed arrangement of the molecules round the axis of $x$, it follows (art. 6 and 9 ,) that $c=c_{1}$, and consequently that when $\theta$ is zero we have $v=v_{n}$. Hence by limiting our view to a spherical and spheroidal wave, both of which emanate from the centre of agitation at the same instant, we perceive that they will constantly cuincide along the axis of $x$. And when $\theta$ is a right angle we have $v_{l}=c_{\mu p}$ which shows that the spherical wave will include, or be included by, the spheroidal wave, accordingly as $c$ is greater or less than $c_{\|}$.

By referring to Professor Airy's Mathematical 'Tracts, p. 346-350, it will be seen that the results obtained in this and the preceding article are sufficient to explain the prin- 
cipal optical phænomena presented by what are called uniaxal crystals. The Professor, following, as I suppose, the method of M. Fresnel, has deduced results similar to these, except that, by his reasoning, the direction of vibration in the spherical waves is the same as we have found it to be in the spheroidal, and the converse. But I apprehend that the 104th and 111 th articles of his valuable tract on this subject are inconsistent with each other; and that the latter, on which the question depends, is erroneous.

I perceive, from the British Association's Report on Physical Optics, that the investigations of M. Cauchy give, for the direction of vibration, the same result as mine; but it appears that his investigations are not founded so immediately as mine upon the physical constitution of the medium.

(13.) The deductions of the last two articles rest upon the supposition (art. 6.) that $v_{l}=s_{i}, v_{l l}=s_{l l}$; but by the equations (2.),

$$
\begin{aligned}
& v_{1}=s_{1} \cdot V\left(1-\frac{s_{1}^{\prime 2}}{s_{1}^{2}} \cdot \frac{4 \pi^{2}}{\lambda_{1}^{8}}+8 c_{0}\right),
\end{aligned}
$$

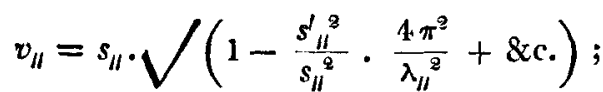

hence these deductions require a corresponding modification. But this is easily effected; for we may suppose the variations of the sums $s^{2}, s_{1}^{2}, s_{\| 1}^{2}, s^{\prime 2}, s_{1}^{\prime 2}, s_{\|}^{\prime 2}$, \&c. depending upon the directions of the coordinates, to be in general small fractions of the sums themselves. Hence, in the last equations, we may regard $\frac{s_{1}^{\prime 3}}{s_{1}^{2}}, \frac{s_{\| 1}^{\prime}}{s_{1 /}^{2}}$ as constant, and (paper at p. 270 of vol. viii.,) equal one to the other; and thus, if we put $\frac{s_{1}^{\prime 2}}{s_{i}^{q}}$ $4 \pi^{2}=A$, we have, instearl of the values of $v$, and $v_{1 /}$ found in articles 9 and 6 ,

$$
\begin{aligned}
& v_{1}=c \cdot \sqrt{\left(1-\frac{\mathrm{A}}{\lambda_{1}^{2}}+8 \mathrm{c} .\right)} \\
& v_{l l}=\sqrt{ }\left(c_{1}^{2} \cos ^{2} \theta+c_{\| l}{ }^{2} \sin ^{2} \theta\right) \cdot \sqrt{ }\left(1-\frac{\mathrm{A}}{\lambda_{\| i}{ }^{2}}+8 \mathrm{c} .\right) .
\end{aligned}
$$

It appears by these equations that the values of $v_{1}$ and $v_{\| \prime}$, and the ratio of one of them to the other, depend, in some measure, upon $\lambda_{l}, \lambda_{l l}$, the lengths of the waves. This is cunfirmed by experience. See Airy's Tracts, p. 354.

(14.) It is well known that light moves through glass, in its ordinary state, with the same velocity in all directions;

Third Series. Vol. 9. No. 56. Dec. 1836. $3 \mathbf{E}$ 
and that the velocity is not affected by any change in the direction of the vibrations: consequently the sums $s^{2}, s_{1}{ }^{2}, \& c$. must, for this medium, be the same whatever be the directions of the coordinates. But it is found, by experiment, that if glass be expanded or contracted in one direction only, it exhibits the same optical phænomena as an uniaxal crystal; the optical axis lying in the direction of the expansion or contraction. (Airy's Tracts, p. 403, art. 178.) Now, it is manifest that since the sums $s^{2}, s_{1}^{2}$, \&c. are originally the same for all directions of the coordinates, these sums must, in the altered state of the glass, be still such that their values will not be affected by turning the coordinates upon an axis taken in the direction of the expansion or contraction; and consequently this experiment affords a verification of our formulæ.

(15.) Whatever be the arrangement of the molecules, we have, by the equations (3.) and the assumption of article (9.),

$$
\begin{aligned}
& v_{1}^{8}=\frac{m}{2} \Sigma \cdot \psi(r) \Delta x^{2} \Delta y^{2}, \\
& v_{\| l}^{2}=\frac{m}{2} \Sigma \cdot \psi(r) \Delta x^{2} \Delta z^{2} ;
\end{aligned}
$$

provided (art. 3.) the directions of $y$ and $z$ are so taken that

$$
\Sigma . \psi(r) \Delta x^{2} \Delta y \Delta z=0 .
$$

Let $x_{1}^{\prime}, y_{1}^{\prime}, z_{l}^{\prime}$ be rectangular coordinates having fixed directions, and the same origin as $x, y, z$; let the axis of $x$ coincide with that of $x_{\prime}^{\prime}$; and let $\theta_{\prime}^{\prime}$ be the angle between $y_{;}^{\prime}$ and $y$ : then

$$
\begin{aligned}
& \Delta x=\Delta x_{l}^{\prime}, \\
& \Delta y=\Delta y_{1}^{\prime} \cos \theta^{\prime}-\Delta z_{i}^{\prime} \sin \theta^{\prime}, \\
& \Delta z=\Delta y \sin \theta^{\prime}+\Delta z_{j}^{\prime} \cos \theta^{\prime} .
\end{aligned}
$$

By substituting these values and, for the sake of abridgrement, putting

we have

$$
\begin{aligned}
& \Sigma . \psi(r) \Delta x^{\prime}{ }^{2} \Delta y^{\prime}{ }^{3}=\sigma, \\
& \Sigma . \psi(r) \Delta x^{\prime}{ }^{\prime} \Delta z^{\prime}{ }^{2}=\sigma^{\prime}, \\
& \Sigma . \psi(r) \Delta x^{\prime}{ }_{1}^{2} \Delta y_{1}^{\prime} \Delta z_{1}^{\prime}=\sigma^{\prime \prime},
\end{aligned}
$$

$v_{l}^{9}=\frac{m}{2} \sigma \cos ^{2} \theta_{i}^{\prime}+\frac{m}{2} \sigma^{\prime} \sin ^{2} \theta_{j}^{\prime}-m \sigma^{\prime \prime} \sin \theta_{i}^{\prime} \cos \theta_{i}^{\prime}$,

$v_{l l}{ }^{2}=\frac{m}{2} \sigma \sin ^{2} \theta_{l}^{\prime}+\frac{m}{2} \sigma^{\prime} \cos ^{2} \theta_{l}^{\prime}+m \sigma^{\prime \prime} \sin \theta_{j}^{\prime} \cos \theta_{j}^{\prime}$,

$\Sigma \cdot \psi(r) \Delta x^{9} \Delta y \Delta z=\left(\sigma-\sigma^{\prime}\right) \sin \theta_{j}^{\prime} \cos \theta_{i}^{\prime}+\sigma^{\prime \prime}\left(\cos ^{2} \theta_{l}^{\prime}-\sin ^{2} \theta_{i}^{\prime}\right.$. 
From these equations we find $\frac{d\left(v_{i}{ }^{2}\right)}{d \theta^{\prime}}=-\frac{d\left(r_{H}{ }^{2}\right)}{d \theta^{\prime}}=-m \Sigma \cdot \psi(r) \Delta x^{2} \Delta y \Delta z:$

so that when

$$
\left(\sigma-\sigma^{\prime}\right) \sin \theta_{i}^{\prime} \cos \theta_{i}^{\prime}+\sigma^{\prime \prime}\left(\cos ^{2} \theta_{i}^{\prime}-\sin ^{2} \theta_{i}^{\prime}\right)=0,
$$

the $\operatorname{sum} \Sigma \cdot \psi(r) \Delta x^{2} \Delta y \Delta z$ is zero as required, while, of the expressions for $v_{1}{ }^{2}$ and $v_{i j}{ }^{2}$, one is a maximum and the other a minimum. The last equation is always possible; for since $\sin \theta_{i}^{\prime} \cos \theta_{i}^{\prime}=\frac{\sin 2 \theta_{i}^{\prime}}{2}$, and $\cos ^{2} \theta_{j}^{\prime}-\sin ^{2} \theta_{i}^{\prime}=\cos 2 \theta_{i}^{\prime}$ it gives

$$
\tan 2 \theta^{\prime}=\frac{2 \sigma^{\prime \prime}}{\sigma^{\prime}-\sigma} \text {. }
$$

(16.) It has been observed (art. 1,) that the sums composed of even products involving odd powers of the differences must, in general, be very small compared with the sums composed of products of the same degree in which the powers of the differences are all even. Lret it then be supposed that $x^{\prime}, y^{\prime}, z^{\prime}$ are rectangular coordinates of which the axes are fixed in the medium; and that the arrangement of the molecules, with respect to these axes, is such that the sums of which the terms involve odd powers of the differences $\Delta x^{\prime}$, $\Delta y^{\prime}, \Delta z^{\prime}$, are either zero (art. 21,) or insensibly small. Let the axis of $x_{1}$ coincide with that of $x^{\prime}$; and let $\theta^{\prime}$ be the angle between $y^{\prime}$ and $y_{l}$; then

$$
\begin{aligned}
& \Delta x_{1}=\Delta x^{\prime}, \\
& \Delta y_{i}=\Delta y^{\prime} \cos \theta^{\prime}-\Delta z^{\prime} \sin \theta^{\prime}, \\
& \Delta z_{i}=\Delta y^{\prime} \sin \theta^{\prime}+\Delta z^{\prime} \cos \theta^{\prime} ;
\end{aligned}
$$

and consequently, when we omit the terms involving the odd powers of the differences $\Delta x^{\prime}, \Delta y^{\prime}, \Delta z^{\prime}$, we have

$$
\Delta x_{1}^{2} \Delta z_{1}^{2}=\Delta x^{\prime 2} \Delta y^{\prime 2} \sin ^{2} \theta^{\prime}+\Delta x^{\prime 2} \Delta z^{12} \cos ^{2} \theta^{\prime} .
$$

For the reasons mentioned in article (9.) we leave out of the expression for $v_{\mu j}{ }^{8}$ (art. 6.) the function $\phi(r)$, and then

$$
\begin{gathered}
v_{l}{ }^{2}=\cos ^{2} \theta \cdot \frac{m}{2} \Sigma \cdot \psi(r) \Delta z_{i}{ }^{2} \Delta x_{i}{ }^{2}+\sin ^{2} \theta \cdot \frac{m}{2} \Sigma \cdot \psi(r) \Delta z_{i}{ }^{2} \Delta y_{l}{ }^{2} \\
-\sin \theta \cos \theta \cdot m \Sigma \cdot \psi(r) \Delta z_{i}{ }^{2} \Delta y_{l} \Delta x_{l} .
\end{gathered}
$$

Now, when the coordinates $x_{1}, y_{1}, z_{1}$ are turned on the common axis of $x_{1}$ and $x^{\prime}$, the sum $\Sigma . \psi(r) \Delta z_{1}{ }^{3} \Delta y_{1}{ }^{2}$ must be of the same value, whether $y_{1}$ coincide with $y^{\prime}$ or $z^{\prime}$; we will therefore suppose it to be sensibly the same for all values of $\theta^{\prime}$ : when, again, we change the coordinates $x_{1}, y_{1}, z_{1}$, for $\bar{x}^{\prime}, y^{\prime}, z^{\prime}$, the last sum in the equation will be composed of terms involving odd powers of $\Delta x^{\prime}$, and will therefore, by 3 E 2 
the supposition, be insensible; hence we shall have, by sub stituting for $\Delta x_{1}^{8} \Delta z_{1}^{2}$ its value previously found,

$$
\begin{aligned}
& v_{\|}^{2}=\cos ^{2} \theta \cdot \frac{m}{2} \Sigma \cdot \psi(r)\left(\Delta \cdot x^{\prime 2} \Delta y^{\prime 2} \sin ^{9} \theta^{\prime}+\Delta x^{\prime 2} \Delta z^{\prime 2} \cos ^{2} \theta^{\prime}\right) \\
& +\sin ^{2} \theta \cdot \frac{m}{2} \Sigma \cdot \psi(r) \Delta z^{\prime 2} \Delta y^{\prime 2} ;
\end{aligned}
$$

or, $\quad v_{1}^{2}=c^{2} \cos ^{2} \sin ^{2} \theta^{\prime}+c^{22} \cos ^{2} \theta \cos ^{2} \theta^{\prime}+c^{\prime \prime 2} \sin ^{2} \theta$;

if, for the sake of abridgement, we put

$$
\begin{gathered}
\frac{m}{z} \Sigma \cdot \psi(r) \Delta x^{\prime 2} \Delta y^{\prime 2}=c, \cdot \frac{m}{2} \Sigma \cdot \psi(r) \Delta x^{\prime 2} \Delta z^{\prime z}=c^{\prime 2}, \\
\frac{m}{2} \Sigma \cdot \psi(r) \Delta y^{\prime 2} \Delta z^{\prime 2}=c^{\prime 2} .
\end{gathered}
$$

(17.) Let $\mathrm{O} x^{\prime}$ (fig. 3,) be the common axis of $x^{\prime}$ and $x_{l} ; \mathrm{O} y^{\prime}$, $\mathrm{O} z^{\prime}, \mathrm{O} y^{\prime}, \mathrm{O} z^{\prime}, \mathrm{O}_{x}, \mathrm{O} y, \mathrm{O} z$, the axes of $y^{\prime}, z^{\prime}, y_{1}, z_{1}, x, y, z$; $y^{\prime} \mathrm{O} y_{1}=\theta^{\prime}$, and $x^{\prime} \mathrm{O} x=\theta$. Now $\mathrm{O} x$ being, by the supposition of art. 6 , in the plane of $x^{\prime} \mathrm{O} y_{1}$, we will suppose $\mathrm{O} y$ to be also in the same plane. Then $y, \mathrm{O} y=x^{\prime} \mathrm{O} x$ $=\theta, \cos x^{\prime} \mathrm{O} y=\sin \theta, \cos y^{\prime} \mathrm{O} y$ $=\cos \theta, \cos \theta^{\prime}, \cos z^{\prime} O y=\cos \theta$ $\sin \theta^{\prime}$; and thus, by the last expression for $v_{l i}^{2}$, we arrive at

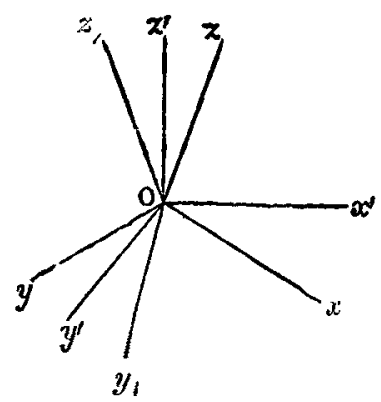

$$
z_{i}^{\prime}=c^{z} \cos ^{2} z^{\prime} \mathrm{O} y+c^{\prime 2} \cos ^{2} y^{\prime} \mathrm{O} y+c^{\prime \prime 2} \cos ^{8} x^{\prime} \mathrm{O} y \text {. }
$$

In deducing this equation we have supposed $\mathrm{O} x$, $\mathrm{O} x$, $O y$, to be in the same plane; but we take for granted that the value of $v^{1 / 2}$ would not be sensibly affected by turning the coordinates upon the axis of $y$; because $v_{\| 1}{ }^{2}=\frac{m}{2} \Sigma \cdot \psi(r) \Delta x^{2} \Delta z^{2}$, and this sum, being symmetrical with respect to $x$ and $z$, retains the same value when $x$ and $z$ are interchanged. Hence it follows that the equation is true in general, and, consequently, that if we change the angles $z^{\prime} \mathrm{O} y, y^{\prime} \mathrm{O} y, x^{\prime} \mathrm{O} y$, for $z^{\prime} \mathrm{O} z, y^{\prime} \mathrm{O} z, x^{\prime} \mathrm{O} z$, it gives also the value of $v_{1}^{2}$.

(18.) It will be remembered that $v_{l l}$ is the velocity of waves moving in the direction of $\mathrm{O} x$, and consisting of vibrations in the direction of $\mathrm{O}_{z}$; and that $v^{\prime}$ is the velocity of waves moving in the same direction, and consisting of vibrations in the direction of $\mathrm{O} y$. The directions of $\mathrm{O} y$ and $\mathrm{O} z$, which determint those of the vibrations must (art. 15.) be so taken 
at right angles to each other and to $\mathrm{O} x$, that of the expressions for $v_{1}^{2}$ and $v_{u}{ }^{2}$, one shall be a maximum, the otlier a minimum.

If the last expression for $v_{1}{ }^{2}$, and the observations subsequently made, be compared with the expression in art. 119, and the observations in articles 120 and 121 of Professor Airy's Tract before quoted, it will be perceived that we have now deduced the findamental laws of M. Fresnel's theory of refraction for biaxal crystals. But the direction of vibration is, as we have previously found in the case of uniaxal crystals, perpendicular to that which this ingenious philosopher supposed. The consequences of these laws have, as it appears from the British Association's Report on Physical Optics, been so ably traced and verified by Sir William Rowan Hamilton and others, that I deem it unnecessary to pursue this part of the theory any further.

$$
\text { I am, Gentlemen, yours, \&c., }
$$

Evesham, June 28, 1836.

\section{Joh N Tovey.}

P.S. In my last paper, vol.viii, p. 501, line 2 from the bottom, for 1 read $\xi$; p. 502, 1. 8 from the bottom, dele comma after $\beta ;$ p. $505,1.11$, for $\frac{a}{x} \operatorname{read} \frac{\alpha}{x}$.

In the valuable paper from M. Cauchy in your last [June] Number, I have noticed the following errors. Vol. viii. p. 4.61, formula (2.), for $a u+b v+c$ read $a u_{1}+b v_{1}+c w_{1}$; and line 5 from bottom, for w read $u$. P. 462 , formula (7.), for $\varepsilon_{n}$ in the denominator read $u_{n}$. P. 463, 1. 13, prefix of; and line 3 from the bottom, for 0 read $a . P .464$, line 14, for $u_{i}$ read $v_{i}$; and 1.15 , for we read We. P. 465, 1. 12, for 19 read 16; and 1.28 , for a w read $a u$. P. 466, 1. 13, for C read $\beta$. P. 467, lines 1 and 3 , for $=v$, in every place, read $=0$; and line 10 dele the first wo.

\section{On Meteoric Stones. By Professor Benzeluus.*}

THE author commences this interesting memoir by consi1 dering which of the conjectures respecting the formation of meteoric stones is the most probable. That which refers these bodies to eruptions of the volcanos of our earth cannot be supported, on account of the distance of the places where they have fallen from any volcano, and also from the different constilution of volcanic products and meteorites; neither can

- From the Journal de Pharmacie for February 1836: communicated by J. D. Smith, Esq., being a translation of an extract, by M. Vallet, from a memoir in Poggendorf's Annalen der Physik und Chemic, vol. xxaiii. p. 1. 